\title{
Elementos culturales visuales que generan identidad en la ciudad de Santa Ana
}

\section{Visual cultural elements that generate identity in the city of Santa Ana}

\section{ISSN 2071-8748 \\ E-ISSN 2218-3345 \\ (c) (9) \\ BY NC SA}

URI: http://hdl.handle.net/11298/977

DOI: https://doi.org/10.5377/entorno.v0i67.7495

\author{
Leida Guadalupe Monterroza-Matute \\ Unasa \\ ORCID:https://orcid.org/0000-0002-6922-143X \\ David Alberto Núñez-Hernández, Unasa \\ david.nunez@unasa.edu.sv \\ Claudia María Estrada-De León, Unasa \\ claudia.deleon@unasa.edu.sv \\ Jaime Josué Martínez, Unasa \\ josuemartininez@unasa.edu.sv \\ Mauricio Alberto Chávez-Núñez, Unasa \\ mauricio.chavez@unasa.edu.sv \\ Universidad Autónoma de Santa Ana
}

Recibido: 20 de octubre 2018 Aprobado: 10 de marzo 2019

\section{Resumen}

Esta investigación cuantitativa describe los elementos culturales visuales que generan identidad en la ciudad de Santa Ana. Realizada en la zona urbana del municipio durante el 2017, con muestra de 734 personas. Permitió identificar información referente a elementos como monumentos, edificaciones, parques y lugares turísticos; de estos, los que poseen mayor representatividad fueron analizados tomando en cuenta los factores sociodemográficos de los encuestados para determinar si estos influyen en la percepción respecto a dichos elementos. También se conoció la opinión de la población santaneca sobre el trabajo que realizan las instituciones encargadas de velar por la promoción y la preservación de la cultura. Los resultados reflejan que el monumento

\section{Abstract}

This quantitative research describes the visual elements that generate cultural identity in the city of Santa Ana. The research was conducted in the urban area of the municipality during 2017, using a sample of 734 people. Information concerning elements such as monuments, buildings, parks and tourist sites was identified; those with greater representation were analyzed taking into consideration the sociodemographic factors of respondents to determine whether they affect the perception regarding such elements. The opinion from the people of Santa Ana in relation to the work performed by institutions responsible for the promotion and preservation of culture was also reflected. The results show that the most representative monument is the 
más representativo es el cañón, la edificación que más los identifica es la catedral, el parque insignia es el Libertad, y el lugar turístico más representativo es el Sihuatehuacán. Los encuestados manifiestan que la Universidad Autónoma de Santa Ana es la institución de educación superior que vela por el fortalecimiento y la promoción de la cultura; y que el Centro de Artes de Occidente es la institución que más realiza esfuerzos en pro de la conservación del arte y la cultura. Esta investigación expone que la catedral es el elemento cultural más representativo para los santanecos, y plantea que es necesario realizar acciones de comunicación encaminadas a resaltar la belleza cultural de la ciudad de Santa Ana tanto a escala nacional como fuera de las fronteras.

\section{Palabras clave}

Identidad cultural-Santa Ana, El Salvadorinvestigaciones; bienes culturales-Santa Ana, El Salvador- investigaciones; patrimonio cultural-Santa Ana, El Salvador- investigaciones; preservación del patrimonio histórico-Santa Ana, El Salvadorinvestigaciones.

\section{Introducción}

EEl patrimonio cultural, de acuerdo con la Organización de las Naciones Unidas para la Educación, la Ciencia y la Cultura (Unesco), "es a la vez un producto y un proceso que suministra a las sociedades un caudal de recursos que se heredan del pasado, se crean en el presente y se transmiten a las generaciones futuras para su beneficio". De ahí la importancia de conocer y preservar esa riqueza.

La cultura visual cobra vital importancia por ser el referente de una identidad social, propia de cada pueblo; y que puede ser transmitida de generación en generación. En efecto, los elementos culturales presentes en cada ciudad representan la razón de su existencia; y su estudio, preservación y divulgación es compromiso de todos los actores sociales.

Sobre la importancia de los distintos elementos que conforman el patrimonio cultural, Llull Peñalba (2005) argumenta que el valor que se les atribuye va más allá de su antigüedad o su estética, dice: "La función referencial de historic cannon; the building that most identifies Santa Ana is the cathedral; the most characteristic park is the Parque Libertad, and the most representative tourist spot is Sihuatehuacán. Respondents stated that Universidad Autónoma de Santa Ana is the higher education institution that ensures the strengthening and promotion of culture, and the Centro de Artes de occidente is the one that makes efforts for the conservation of art and culture. This research states that the cathedral is-among all monuments and places-the most representative cultural element for the people of Santa Ana, and asserts the need to improve the communication process aimed at highlighting the cultural beauty of the city of Santa Ana, both nationally and outside borders.

\section{Keywords}

Cultural identity-Santa Ana, El Salvador-research; cultural property-Santa Ana, El Salvador research; cultural heritage-Santa Ana, El Salvador research; historical heritage preservation-Santa Ana, El Salvadorresearch.

los bienes culturales influye en la percepción del destino histórico de cada comunidad, en sus sentimientos de identidad nacional" (p. 181).

García (1998) opina que "una idea clave en la legislación sobre el patrimonio es la de su relación con la identidad. La asociación entre identidad y patrimonio, a nivel temático es una constante legislativa, si bien no siempre se explicita conceptualmente esa relación"; y hace referencia a la ley española sobre patrimonio, en donde no se menciona el concepto de identidad. Por el contrario, la legislación salvadoreña sobre patrimonio cultural sí hace alusión a la identidad al afirmar que "Ios bienes culturales, expresan las tradiciones de nuestro pueblo y que configuran el fundamento y razón de ser de la identidad e idiosincrasia de los salvadoreños" (II Considerando de la Ley Especial de Protección al Patrimonio Cultural de El Salvador).

En la actualidad, Santa Ana es considerada una de las principales ciudades de El Salvador por su extensión territorial, su cantidad de habitantes, su agricultura, su 
riqueza cultural y otra serie de elementos que la distingue de otros lugares del país.

La riqueza cultural tangible que posee Santa Ana se evidencia en sus calles y avenidas; su centro histórico es considerado de los más representativos del país debido a la gran cantidad de edificaciones de connotada arquitectura en un mismo lugar, además posee parques con diferentes elementos que generan identidad a la población como resultado de costumbres y tradiciones o aspectos socioculturales, asimismo, existen una serie de monumentos ubicados en diversos puntos de la ciudad que ofrecen un testimonio histórico, entre ellos se pueden mencionar El Casino Santaneco, El Cañón, Catedral de Santa Ana, Parque Colón, El Teatro Nacional, entre otros.

Por lo general, existe una tendencia a intuir que la población de Santa Ana reconoce cada elemento cultural y conoce además cuáles son los acontecimientos o hechos en torno a ellos. Pero es de suma importancia pasar de una simple suposición a contar con información fehaciente que dé con certeza los datos que determinen cuál es la percepción de la población de Santa Ana sobre los diferentes elementos culturales visuales. A partir de ahí, las diferentes instituciones que promueven la cultura en la ciudad pueden también implementar planes de trabajo en relación con lo que realmente la población piensa.

Caraballo (2011) y Cruces (1998) citados por Meca Ospina (2016) afirman que:

el valor patrimonial de un bien cultural se asigna socialmente, por lo tanto, es temporalmente mutable y está cargado de lecturas múltiples e incluso contradictorias. Esto significa que el valor cultural de una manifestación o bien, seguramente, en otro momento histórico, tuvo significado diferente e incluso opuesto al que hoy se le asigna. Los referentes de valor cambian, por lo tanto, hay que tener en cuenta que los valores atribuidos localmente serán seguramente mucho más amplios y complejos que su valor asignado por los criterios académicos o desde las instancias públicas centrales (p. 31).

\section{Metodología}

La investigación fue de tipo cuantitativo con enfoque descriptivo. Para determinar el tamaño de la muestra, se utilizó la fórmula para poblaciones finitas y la base a datos de la estimación y proyección de población municipal de El Salvador, la cual estipulaba una población de 272.554 personas, para el municipio de Santa Ana, en el año 2017. El error muestral fue de $+-4 \%$ y un nivel de confianza de 97 $\%$. Se realizó un muestreo probabilístico simple, en donde las unidades de muestreo fueron seleccionadas de forma aleatoria para garantizar que todos los elementos de la población tuvieran la misma posibilidad de ser escogidos. Abad y Servín (1982) argumentan que "extraer una muestra aleatoria simple consistente de $n$ unidades elegidas de entre $N$ de que consta la población, es extraerlas de manera que a todas y a cada una de las $\left(\frac{N}{n}\right)$ muestras posibles se les asigne y respete una probabilidad igual de ser elegidas" (p. 41).

\section{Recolección de datos}

Para la recolección de datos, se utilizó la técnica de encuesta cara a cara, y un cuestionario con 24 preguntas cerradas, el cual fue aplicado en el mes de julio de 2017. El levantamiento muestral se realizó fuera del centro histórico de la ciudad de Santa Ana para evitar un sesgo en la investigación, por la cercanía visual de los elementos culturales ahí presentes.

\section{Análisis de los datos}

La información recolectada mediante la encuesta fue procesada en el programa Excel; y los resultados se analizaron de acuerdo con dos variables:

- Lugar más representativo de la ciudad de Santa Ana

- Razón por la cual se considera el lugar más representativo

Los resultados obtenidos fueron categorizados de acuerdo con la Ley Especial al Patrimonio Cultural de El Salvador (1993), la cual establece en el artículo 3, inciso ñ, párrafo 1 , que "se consideran, además, como bienes culturales todos aquellos monumentos de carácter arquitectónico, escultórico, urbano, jardines históricos, plazas, conjuntos históricos, vernáculos y etnográficos, centros históricos, sitios históricos y zonas arqueológicas". En ese sentido, para efectos de la investigación, se clasificaron los elementos culturales visuales en edificaciones, monumentos, parques y lugares turísticos.

\section{Resultados}

LSegún los resultados, independientemente de la clasificación de patrimonio, el referente a la ciudad de Santa 
Ana está bien señalado por la población, siendo la catedral de Nuestra Señora de Santa Ana, o simplemente catedral de Santa Ana, la más reconocida por la población. En segundo lugar, los entrevistados mencionaron el Teatro de Santa Ana. En la categoría Otros, se agruparon elementos culturales que obtuvieron un porcentaje de mención inferior al 5 \%, como la alcaldía municipal de Santa Ana, el parque Libertad, El Cañón y el Casino Santaneco, entre otros (ver figura 1).

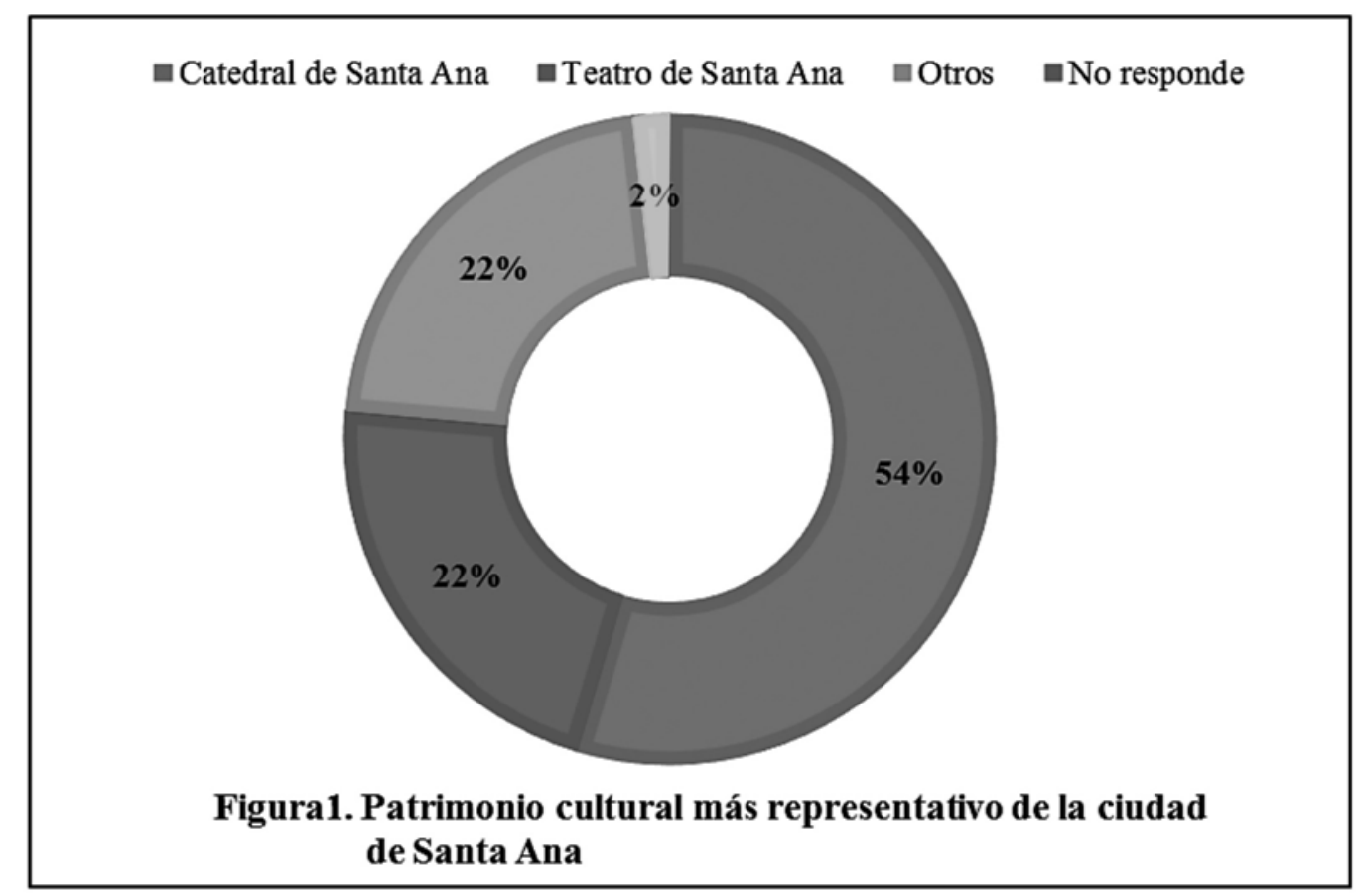

En cuanto a la clasificación de los elementos culturales, la investigación exploró cuál es la edificación, el monumento, parque y lugar turístico más representativo de la ciudad, encontrando que nuevamente la catedral fue señalada como la más notoria de la ciudad $(70,6 \%)$, pero en este caso como la edificación con la que más personas se identifican.

Con respecto al monumento que genera un mayor grado de identidad entre los pobladores, el 27,7 \% nombró El Cañón; $26,3 \%$ el monumento a La Libertad y para el $24,7 \%$ de los entrevistados fue el monumento a Monseñor Romero el más representativo de Santa Ana. Los resultados también arrojaron que el parque más representativo de la ciudad es el Parque Libertad (75,5 \%) y el lugar turístico fue el Sihuatehuacán (69,9\%).

Al preguntar por la razón por la que el elemento cultural mencionado es considerado el más representativo de la ciudad, independientemente de la clasificación (edificación, monumento, parque y lugar turístico), la respuesta que obtuvo mayor porcentaje en todas las categorías fue "Por historia", lo que denota el valor que tiene el componente histórico en la valoración del patrimonio cultural. 
Tabla 1

\section{Elemento cultural más representativo y razón principal por la que es más representativo}

\begin{tabular}{ll}
\hline Elemento cultural & Importancia de la historia (\%) \\
\hline Edificación: Catedral de Santa Ana & 84,3 \\
Monumento: El Cañón & 83,3 \\
Parque: Libertad & 71,7 \\
Lugar turístico: Sihuatehuacán & 68,2 \\
\hline
\end{tabular}

\footnotetext{
Nota: Los porcentajes representan a la cantidad de encuestados que respondieron "la historia" como razón para ser la edificación, monumento, parque y lugar turístico más representativo.

Fuente: elaborada por los autores.
}

Sobre el acceso a información que la población ha tenido sobre monumentos culturales o temas relacionados a la cultura de la ciudad, una leve mayoría expresa que "Si" ha recibido (52.9\%), pero un porcentaje significativo del $47.1 \%$, expresó no haber tenido acceso a dicha información.

\section{Discusión}

Esta investigación evidenció que el patrimonio cultural de la ciudad de Santa Ana puede enmarcarse en dos grandes monumentos. Inicialmente, la población señala a la Catedral de Santa Ana, seguido del Teatro Nacional de Santa Ana como los principales íconos culturales de la ciudad. Sin embargo existen otras edificaciones con un gran valor histórico y un alto potencial turístico, que no son reconocidos por la población, tales como el edificio de la Alcaldía, el Casino Santaneco y el Estadio Óscar Alberto Quiteño.

En todas las categorías presentadas al entrevistado, se le señalaron diferentes razones por las cuales podía escoger cuál, a su criterio, era la razón principal por la que los diferentes patrimonios gozan de reconocimiento y, por ende, producen en los pobladores de la ciudad de Santa Ana una identidad. Luego del cruce de variables, la respuesta encontrada fue que la historia es la razón que tiene un peso significativo para la elección entre los pobladores frente a otras razones, como la ubicación del elemento cultural y su arquitectura o diseño, que no superan el $10 \%$, lo cual confirma la importancia del referente histórico del patrimonio cultural.

Sobre el poder extraordinario que poseen los objetos patrimoniales y su relación con la historia, Santacana (2014) afirma lo siguiente:
El objeto es un talismán del pasado en la medida que existen teorías a su alrededor; sin ellas, sin la historia que le da soporte, se transforma en una curiosidad o en un elemento carente de todo valor. Cuando los objetos pierden los relatos en los que se apoyan, se transforman en elementos de simple entretenimiento. (p. 46).

Los elementos culturales que están en la ciudad de Santa Ana poseen una historia que los sustenta y les permite mantenerse a través del tiempo; les otorgan credibilidad $y$, por ende, se tornan en reconocidos patrimonios culturales que se conservan aún con el paso del tiempo.

La identidad cultural parte de la interacción directa entre las personas y los diferentes objetos que se encuentran en su entorno. Primeramente se habla de una relación de conocimiento con dichos objetos; y poco a poco, con el paso del tiempo, estos se van transformando en elementos que generan identidad cultural.

Los elementos señalados como los más representativos, indistintamente de su antigüedad, poseen un valor histórico grande, tanto que les permite sobresalir de otros a pesar de la ubicación en la que se encuentren y la difusión que se les realiza.

Con esta investigación se evidencia que las instituciones encargadas de promover la cultura santaneca, a pesar de realizar diversas actividades, presentaciones y otro tipo de exposiciones culturales aún no han logrado impactar a la mayoría de la población, les falta hacer el esfuerzo para que los habitantes de la ciudad conozcan sobre su historia y se fortalezca la promoción de la cultura local. 
Citando a Pastor (2003), los beneficios de promover el patrimonio cultural de una ciudad, se reflejan en dos sentidos, para el turista, ya que conoce aspectos que forman parte de la identidad de la ciudad visitada y para el anfitrión pues se preocupa por "activar y preservar elementos que, en otras circunstancias podrían quedar relegados" (p. 99) por tanto en la medida que los habitantes de la ciudad conozcan sobre su riqueza cultural habrá mayor conciencia para preservar los bienes patrimoniales y para difundirlos o promocionarlos, ya sea local o internacionalmente

\section{Conclusiones}

- La población santaneca identifica los elementos culturales más reconocidos de Santa Ana; su ubicación y mayormente la tradición oral son determinantes al momento de decidir la representatividad, aunque no necesariamente esa identificación se relacione a los factores sociodemográficos de la población.

- El patrimonio cultural visual más reconocido por la población santaneca independientemente de su clasificación es Catedral de Santa Ana.

- Es necesario brindar información sobre otros elementos culturales visuales que no se mencionan como principales, ya que sí son muy importantes y poseen gran riqueza e historia, pero por falta de conocimiento los santanecos no los mencionan. Al realizar campañas de información u otro tipo de elemento comunicacional, se debe culturizar sobre la historia y trascendencia de la variedad de elementos culturales, no solo los más reconocidos, potenciando el valor de aquellos de los cuales la población ignora su gran relevancia.

- Las instituciones que trabajan en pro de la cultura, al igual que las universidades, deben fortalecer sus planes de trabajo y dar seguimiento permanente y adecuado a sus programas culturales para lograr un mejor impacto en la población.

\section{Referencias}

BAbad, A., y Servín, L.A. (1982). Introducción al muestreo. (2a ed.). México D. F.: Limusa.

Asamblea Legislativa. (1993). Ley Especial al Patrimonio Cultural de El Salvador. Recuperado de https://www. asamblea.gob.sv/decretos/details/235

García, J.L. (1998). De la cultura como patrimonio al patrimonio cultural. Recuperado de https:// revistas.ucm.es/index.php/POSO/article/download/ POSO9898130009A/25044

Llull Peñalba, J. (2005). Evolución del concepto y de la significación social del patrimonio cultural. Arte, individuo y sociedad. Recuperado de http:// revistas.ucm.es/index.php/ARIS/article/view/ ARIS0505110177A/5813

Meca Ospina, J (2016). Patrimonio cultural: ¿espacio de imaginación o de especulación? En Carreño Hernández, C.A., Meca Ospina, J., Mora Salinas, M.J., Ochoa Espitia, K.P., y Rodríguez Gutiérrez, L.M. (Eds), Cuadernos de patrimonio cultural. Reflexiones contemporáneas. pp. 27-43. Recuperado de http://www.uptc.edu.co/export/ sites/default/facultades/f_educacion/maestria/patrim _cultural/inf_adicional/documentos/cuadernos_patri_ cultural.pdf

Organización de las Naciones Unidas para la Educación, la Ciencia y la Cultura (2014). Indicadores UNESCO de Cultura para el desarrollo: Manual metodológico. Recuperado de http://es.unesco.org/creativity/sites/ creativity/files/iucd_manual_metodologico_1.pdf

Pastor, M. J. (octubre, 2003). El patrimonio cultural como opción turística. Horizontes antropológicos. Porto Alegre, año 9, N. 20, pp. 97-115. Recuperado de: http:// www.scielo.br/pdf/ha/v9n20/v9n20a05.pdf

Santacana, M. J. (2014). Patrimonio, educación e historia: El poder invisible del pasado. En Prats, J., Barca, I., y López Facal, R. (Eds.), Historia e identidades culturales. pp. 41-50. Recuperado de http://www. ub.edu/histodidactica/images/documentos/pdf/ Identidades\%20Culturales.pdf 